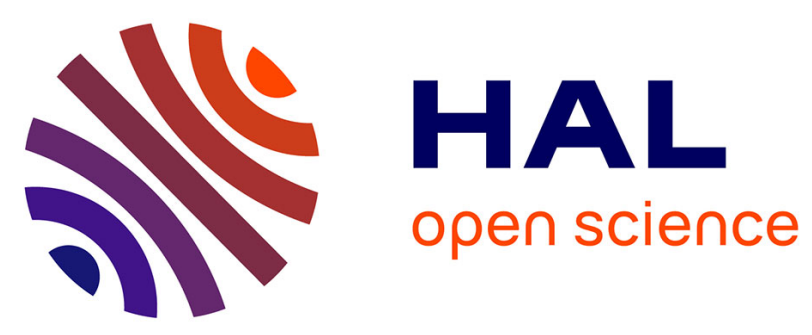

\title{
THE NUMBER OF LIMIT CYCLES BIFURCATING FROM THE PERIOD ANNULUS OF QUASI-HOMOGENEOUS HAMILTONIAN SYSTEMS AT ANY ORDER
}

\author{
Jean-Pierre Françoise, Hongjin He, Dongmei Xiao
}

\section{To cite this version:}

Jean-Pierre Françoise, Hongjin He, Dongmei Xiao. THE NUMBER OF LIMIT CYCLES BIFURCATING FROM THE PERIOD ANNULUS OF QUASI-HOMOGENEOUS HAMILTONIAN SYSTEMS AT ANY ORDER. Journal of Differential Equations, 2021, 276, pp.318-341. 10.1016/j.jde.2020.12.015 . hal-03146819

\section{HAL Id: hal-03146819 \\ https://hal.sorbonne-universite.fr/hal-03146819}

Submitted on 19 Feb 2021

HAL is a multi-disciplinary open access archive for the deposit and dissemination of scientific research documents, whether they are published or not. The documents may come from teaching and research institutions in France or abroad, or from public or private research centers.
L'archive ouverte pluridisciplinaire HAL, est destinée au dépôt et à la diffusion de documents scientifiques de niveau recherche, publiés ou non, émanant des établissements d'enseignement et de recherche français ou étrangers, des laboratoires publics ou privés. 


\title{
THE NUMBER OF LIMIT CYCLES BIFURCATING FROM THE PERIOD ANNULUS OF QUASI-HOMOGENEOUS HAMILTONIAN SYSTEMS AT ANY ORDER
}

\author{
JEAN-PIERRE FRANÇOISE ${ }^{1}$, HONGJING HE ${ }^{2}$ AND DONGMEI XIAO ${ }^{2 \dagger}$
}

\begin{abstract}
A necessary and sufficient condition is given for quasihomogeneous polynomial Hamiltonian systems having a center. Then it is shown that there exists a bound on the number of limit cycles bifurcating from the period annulus of quasi-homogeneous Hamiltonian systems at any order of Melnikov functions; and the explicit expression of this bound is given in terms of $\left(n, k, s_{1}, s_{2}\right)$, where $n$ is the degree of perturbation polynomials, $k$ is the order of the first nonzero higher order Melnikov function, and $\left(s_{1}, s_{2}\right)$ is the weight exponent of quasi-homogeneous Hamiltonian with center. This extends some known results and solves the Arnol'dHilbert's 16th problem for the perturbations of homogeneous or quasi-homogeneous polynomial Hamiltonian systems.
\end{abstract}

\section{INTRODUCTION}

In this article, we study the bifurcation problem of limit cycles of a polynomial Hamiltonian system with center under a small polynomial perturbation:

$$
\begin{aligned}
& \frac{d x(t)}{d t}=-\frac{\partial H(x, y)}{\partial y}+\epsilon p(x, y) \\
& \frac{d y(t)}{d t}=\frac{\partial H(x, y)}{\partial x}+\epsilon q(x, y)
\end{aligned}
$$

where $|\epsilon|<<1, H(x, y)$ is a polynomial of degree $m$ and the associated Hamiltonian system has a center at the origin $O(0,0)$ (note that Euler's

2010 Mathematics Subject Classification. 34C07, 37G15, 34C08.

Key words and phrases. Quasi-homogeneous Hamiltonian systems, global center, higher order Melnikov functions, limit cycles.

$\dagger$ The corresponding author. The third author was partially supported by Innovation Program of Shanghai Municipal Education Commission and the National Natural Science Foundations of China number 11931016. 
identity implies then that $H(0,0)=0), p(x, y)$ and $q(x, y)$ are real polynomials with degree at most $n$.

Suppose $\gamma_{h}$ is a closed orbit around the center $O(0,0)$ of the Hamiltonian system

$$
\begin{aligned}
& \frac{d x(t)}{d t}=-\frac{\partial H(x, y)}{\partial y} \\
& \frac{d y(t)}{d t}=\frac{\partial H(x, y)}{\partial x}
\end{aligned}
$$

Then $\gamma_{h}$ is an oval of the level set

$$
\{(x, y): H(x, y)=h, \text { for a real number } h\} .
$$

Without loss of generality, we assume that $H(x, y)>0$ on the closed orbit $\gamma_{h}$. Then there exists a positive number $h_{1}$ such that the set $\left\{(x, y): H(x, y)=h, 0<h<h_{1}\right\}$ is the maximum period annulus which contains closed orbits $\gamma_{h}$ of system (2), where $h_{1}$ may be $+\infty$. Let

$$
U=\bigcup_{0<h<h_{1}} \gamma_{h}
$$

be a period annulus of system (2). To study the bifurcation problem of limit cycles at any order $k$ from the period annulus $U$ for system (1) as $\epsilon \neq 0$, a basic way is to discuss the Poincaré map defined on the period annulus $U$ (cf. $[2-4,6]$ ), which can be expressed on as

$$
P(h, \epsilon)=h+\epsilon^{k} M_{k}(h)+\epsilon^{k+1} M_{k+1}(h)+\ldots,
$$

where $M_{k}(h) \not \equiv 0$ as $0<h<h_{1}$, and $M_{k}(h)$ is called $k$ th order Melnikov function or also the bifurcation function (cf. [4]). Poincaré-PontryaginMelnikov theorem shows the relationship between the number of limit cycles of system (1) and the number of isolated zeros of $M_{k}(h)$ on the interval $\left(0, h_{1}\right)$; that is, a simple zero of $M_{k}(h)$ corresponds to a limit cycle. The weaken Hilbert 16th problem proposed by Arnol'd is to give a bound on the number of isolated zeros of the first order Melnikov function. The Arnol'd-Hilbert's 16th problem is to give a bound on the number of limit cycles bifurcating at any order $k$ Melnikov functions from the period annulus $U$ for system (1) as $|\epsilon| \ll 1$ (cf. [4]).

A general perturbation theory and the computation of higher order Melnikov functions were developed in $[2-4,6,12,13,17]$. However, it is still challenging to give the explicit bound on the number of limit cycles bifurcating from the period annulus $U$ of polynomial Hamiltonian systems at any order $k$. To our knowledge, there are few results on the explicit bound (cf. $[6-8,16])$. We list some of them. Iliev in $[9]$ 
considered the case of the perturbations of the harmonic oscillator, $H=\frac{1}{2}\left(x^{2}+y^{2}\right)$, and Buica et al. in [1] considered the perturbations of $H=\left(x^{2}+y^{2}\right)^{m}$ with $m \geq 2$, and they obtained the explicit bound on the number of limit cycles bifurcating from the period annulus $U$ of the two classes of Hamiltonian systems at any order $k$. Note that those Hamiltonian functions are homogeneous polynomials.

In this article, we first characterize the $\left(s_{1}, s_{2}\right)$-quasi-homogeneous polynomial Hamiltonian systems having a center. Then we build explicitly the Gelfand-Leray derivative and the algorithm of the successive derivatives for system (1) with homogeneous polynomial Hamiltonian. This helps us to extend the perturbation theory to $\left(s_{1}, s_{2}\right)$-quasihomogeneous polynomial Hamiltonians; where the Euler's identity for homogeneous is replaced by a more general condition that there exists a linear vector field $C=s_{1} x \frac{\partial}{\partial x}+s_{2} y \frac{\partial}{\partial y}$ with positive integers $s_{1}, s_{2}$ such that:

$$
C . H=s_{1} x \frac{\partial H}{\partial x}+s_{2} y \frac{\partial H}{\partial y}=m H .
$$

Further, we provide an explicit bound (depending on $k, n, s_{1}$ and $s_{2}$ ) on the number of limit cycles bifurcating at any order $k$ from the period annulus $U$ of $\left(s_{1}, s_{2}\right)$-quasi-homogeneous polynomial Hamiltonian systems, which extends two theorems of Iliev and Buica et al. (cf. [9] [1]). However, to our knowledge, this upper bounds are not the optimal ones, see our example. Note that the existence of a bound for the maximal order $k$ of the bifurcation function $M_{k}(h)$ (called Iliev number in [4]) implies the existence of a bound for the number of limit cycles in the case where the perturbation setting displays a Bautin ideal. The existence of this bound was recently proved in the article [4]. Hence, our result solves the Arnol'd-Hilbert's 16th problem for these cases of perturbations of homogeneous or quasi-homogeneous polynomial Hamiltonian systems.

The rest of this article is organized as follows. In section 2, the homogeneous or quasi-homogeneous polynomial Hamiltonian systems having center are characterized. In section 3, we illustrate the construction of higher-order Melnikov functions and give an upper bound on the number of isolated zeros of $M_{k}(h)$ in the interval $\left(0, h_{1}\right)$ for the perturbation system (1) with homogeneous polynomial Hamiltonians. In section 4, we improve the upper bound on the number of isolated zeros of $M_{k}(h)$ in the interval $(0,+\infty)$ by using the Liapunov $\left(s_{1}, s_{2}\right)$ trigonometric functions for $\left(s_{1}, s_{2}\right)$ - quasi-homogeneous systems, and we give an example to show the upper bounds obtained in this paper are not the optimal ones. A general discussion on our methods are 
given in the last section. It shows the method can deal with the more general case, that is, there exists a polynomial $m(H)$ explicitly known and polynomials $(f, g) \in \mathbb{K}[x, y]$, so that $m(H)=f \frac{\partial H}{\partial x}+g \frac{\partial H}{\partial y}$, where $\mathbb{K}[x, y]$ is polynomial ring on the number field $\mathbb{K}, \mathbb{K}=\mathbb{R}, \mathbb{C}$.

\section{The Homogeneous or QUASI-Homogeneous POlynOMIAL HAMILTONIAN SYSTEMS WITH A CENTER}

In this section we characterize homogeneous or quasi-Homogeneous Hamiltonian systems having a center. If the system has a center, then the center is at the origin $O(0,0)$ and it is global, that is, $\mathbb{R}^{2} \backslash\{O\}$ is filled by periodic orbits. Note that Gavrilov, Giné and Grau (cf. [8]) constructed families of non-Hamiltonian quasi-homogeneous centers. Let us first recall definitions of quasi-Homogeneous polynomial Hamiltonian $H(x, y)$ (cf. [14]).

A function $H(x, y)$ is called $\left(s_{1}, s_{2}\right)$-quasi-homogeneous with weight degree $m$ if there exist positive integers $s_{1}, s_{2}$ and $m$ such that

$$
H\left(\lambda^{s_{1}} x, \lambda^{s_{2}} y\right)=\lambda^{m} H(x, y), \quad \forall \lambda>0,
$$

where $\left(s_{1}, s_{2}\right)$ is called the weight exponent and $m$ is called weight degree of this quasi-homogeneous function $H(x, y)$.

If $s_{1}=s_{2}=1$, then the function $H(x, y)$ is called $m$ th homogeneous or homogeneous with degree $m$. If $s_{1} \neq s_{2}$, without loss of generality we can assume that $s_{1}$ and $s_{2}$ are coprime and $s_{1}<s_{2}$. Indeed, if $s_{1}=l s_{1}^{\prime}$ and $s_{2}=l s_{2}^{\prime}$ with coprime $s_{j}^{\prime}, j=1,2$, then taking $\lambda^{\prime}=\lambda^{l}$ and $m^{\prime}=l m$ in formula (4) above, one obtains formula $\left(4^{\prime}\right)$ with prime over the parameters. Removing the prime in this "new" formula we have that either $s_{1}=s_{2}=1$ (the homogeneous case) or $s_{1}<s_{2}$ are co-prime (the quasi-homogeneous case).

The following lemma shows an equivalent definition of the quasihomogeneous functions.

Lemma 1. Assume that $H(x, y)$ is $C^{1}$ function. Then $H(x, y)$ is a $\left(s_{1}, s_{2}\right)$-quasi-homogeneous function of weight degree $m$ if and only if $C . H=m H$, where $C=s_{1} x \frac{\partial}{\partial x}+s_{2} y \frac{\partial}{\partial y}$.

In the next theorem we characterize all quasi-homogeneous polynomial Hamiltonian systems having centers. 
Theorem 2. Assume that $H(x, y)$ is an $\left(s_{1}, s_{2}\right)$-quasi-homogeneous polynomial of weight degree $m$. Then Hamiltonian system (2) has a center at the origin $O(0,0)$ if and only if the following conditions hold:

(a) $s_{1} s_{2} \mid m$, that is, $s_{1} s_{2}$ divides $m$;

(b) $H^{*}(x, y)=H\left(x^{\frac{1}{s_{2}}}, y^{\frac{1}{s_{1}}}\right)$ is a homogenous polynomial of degree $\frac{m}{s_{1} s_{2}}$;

(c) the homogeneous polynomial $H^{*}(x, y)$ has no real linear factors, that is, $H^{*}(x, y)=f_{1} f_{2} \ldots f_{s}$, where $f_{1}, f_{2}, \ldots, f_{s}$ are irreducible homogenous polynomials of degree even $\left(f_{i}, f_{j}\right.$ can be the same with different $i, j$ ).

Moreover, the center is global if it exists. Hence, without loss of generality, we assume that the level set $\gamma_{h}=\{(x, y): H(x, y)=h\}$ is an oval around $O(0,0)$ for any $0<h<+\infty$.

When $s_{1}=s_{2}=1$, from Theorem 2 we directly obtain the necessary and sufficient conditions for the $m$ th homogeneous polynomial Hamiltonian system having center as follows.

Corollary 3. Assume that $H(x, y)$ is an mth homogeneous polynomial. Then system (2) has a center at the origin $O(0,0)$ if and only if $H(x, y)$ has no real linear factors. Moreover, the center is global if it exists. Hence, without loss of generality, $\gamma_{h}=\{(x, y): H(x, y)=$ $h$, for a real $h \in(0,+\infty)\}$ are ovals around the center.

To prove Theorem 2, we first show some basic properties of quasihomogenous polynomials.

Proposition 4. $H(x, y)$ is an $\left(s_{1}, s_{2}\right)$-quasi-homogeneous polynomial of weight degree $m$ if and only if the power vector $\left(m_{1}, m_{2}\right)$ of every monomial $x^{m_{1}} y^{m_{2}}$ of $H(x, y)$ satisfies $s_{1} m_{1}+s_{2} m_{2}=m$.

Proof. If $H(x, y)$ is an $\left(s_{1}, s_{2}\right)$-quasi-homogeneous polynomial of weight degree $m$, then by definition, we know that the power vector $\left(m_{1}, m_{2}\right)$ of every monomial $x^{m_{1}} y^{m_{2}}$ of $H(x, y)$ satisfies

$$
\left(\lambda^{s_{1}} x\right)^{m_{1}}\left(\lambda^{s_{2}} y\right)^{m_{2}}=\lambda^{m} x^{m_{1}} y^{m_{2}} .
$$

Thus, $s_{1} m_{1}+s_{2} m_{2}=m$. So the necessity is proved.

We now prove the sufficiency. Assume that the power vector $\left(m_{1}, m_{2}\right)$ of every monomial $x^{m_{1}} y^{m_{2}}$ of $H(x, y)$ satisfies $s_{1} m_{1}+s_{2} m_{2}=m$. Then we have $\left(\lambda^{s_{1}} x\right)^{m_{1}}\left(\lambda^{s_{2}} y\right)^{m_{2}}=\lambda^{m} x^{m_{1}} y^{m_{2}}$ for every monomial of $H(x, y)$. Therefore the polynomial $H(x, y)$ satisfies (4). This completes the proof. 
A monomial $x^{n_{1}} y^{n_{2}}$ is called the monomial of the polynomial $H(x, y)$ with lowest degree if $n_{1}+n_{2} \leq m_{1}+m_{2}$ for all monomial $x^{m_{1}} y^{m_{2}}$ of $H(x, y)$, and

$$
n_{2}<m_{2} \quad \text { if } \quad n_{1}+n_{2}=m_{1}+m_{2} .
$$

Proposition 5. If the polynomial $H(x, y)$ is $\left(s_{1}, s_{2}\right)$-quasi-homogeneous with weight degree $m$, then for the power vector $\left(m_{1}, m_{2}\right)$ of every monomial $x^{m_{1}} y^{m_{2}}$ of the polynomial $H(x, y)$, there exists an integer $k \geq 0$ such that

$$
m_{1}=n_{1}-k s_{2}, \quad m_{2}=n_{2}+k s_{1},
$$

where $\left(n_{1}, n_{2}\right)$ is the power vector of the monomial of the polynomial $H(x, y)$ with lowest degree.

Proof. Consider a monomial $x^{m_{1}} y^{m_{2}}$ of the quasi-homogenous polynomial $H(x, y)$, and define $A$ and $B$ through

$$
m_{1}=n_{1}+A, \quad m_{2}=n_{2}+B .
$$

From Proposition 4 we have

$$
s_{1} m_{1}+s_{2} m_{2}=m, s_{1} n_{1}+s_{2} n_{2}=m .
$$

From (6) and (7), we obtain that $s_{1} A+s_{2} B=0$. Since $s_{1}$ and $s_{2}$ are coprime, so there exists an integer $k$ such that

$$
A=-k s_{2}, \quad B=k s_{1} .
$$

Since $x^{n_{1}} y^{n_{2}}$ is the monomial of the polynomial $H(x, y)$ with the lowest degree, we have

$$
m_{1}+m_{2}=n_{1}+n_{2}+k\left(s_{1}-s_{2}\right) \geq n_{1}+n_{2} .
$$

So $k \geq 0$ when $s_{1}>s_{2}$. If $s_{1}=s_{2}$, by the definition of $x^{n_{1}} y^{n_{2}}$, $m_{2}=n_{2}+k s_{1} \geq n_{2}$. It also shows $k \geq 0$. Substituting (8) into (6) the proposition follows.

Proposition 6. Assume that an $\left(s_{1}, s_{2}\right)$-quasi-homogeneous polynomial of degree $m$ has no real linear factors, then

(a) $s_{1} s_{2} \mid m$, that is $s_{1} s_{2}$ divides $m$;

(b) $H^{*}(x, y)=H\left(x^{\frac{1}{s_{2}}}, y^{\frac{1}{s_{1}}}\right)$, which is a homogenous polynomial of degree $\frac{m}{s_{1} s_{2}}$;

Proof. Let $x^{n_{1}} y^{n_{2}}$ be the monomial of the quasi-homogeneous polynomial $H(x, y)$ with lowest degree. Since $H(x, y)$ has no linear factors, $n_{2}=0$ by proposition 5 . 
Thus, there exist a positive integer $k^{*}$ and a monomial $x^{m_{1}} y^{m_{2}}$ with $m_{1}=0$ such that $m_{1}=n_{1}-k^{*} s_{2}=0$ and $m_{2}=k^{*} s_{1}$. So $n_{1}=k^{*} s_{2}$.

From (6) and (8), every monomial $x^{m_{1}} y^{m_{2}}$ of $H(x, y)$ satisfies that $m_{1}=\left(k^{*}-k\right) s_{2}$ and $m_{2}=k s_{1}$ for some $k \geq 0$. Therefore, we have $x^{m_{1}} y^{m_{2}}=\left(x^{s_{2}}\right)^{k^{*}-k}\left(y^{s_{1}}\right)^{k}$ and

$$
s_{1} m_{1}+s_{2} m_{2}=s_{1} s_{2}\left(k^{*}-k\right)+s_{2} s_{1} k=m
$$

by $H(x, y)$ being a $\left(s_{1}, s_{2}\right)$-quasi-homogeneous polynomial of degree $m$. Hence, $s_{1} s_{2} k^{*}=m$, which implies the conclusion (a) and $H^{*}(x, y)=$ $H\left(x^{\frac{1}{s_{2}}}, y^{\frac{1}{s_{1}}}\right)$, which is a homogenous polynomial of degree $k^{*}=\frac{m}{s_{1} s_{2}}$, that is the conclusion (b).

We are now in position to prove Theorem 2.

Proof of Theorem 2. Let $H(x, y)$ be an $\left(s_{1}, s_{2}\right)$-quasi-homogeneous polynomial of weight degree $m$. Assume that the Hamiltonian system (2) with Hamiltonian $H(x, y)$ has a center at $O(0,0)$. Then from the page 136 of [14], the polynomial $H=H(x, y)$ cannot have real linear factors because

$$
m H=s_{1} x \frac{\partial H}{\partial x}+s_{2} y \frac{\partial H}{\partial y} .
$$

Therefore, conditions (a) and (b) follow from Proposition 6.

We now prove the conclusion (c) by contradiction.

Assume that the homogeneous polynomial $H^{*}(x, y)$ has a real linear factor $a x+b y$, where $a^{2}+b^{2} \neq 0$. Since $H(x, y)=H^{*}\left(x^{s_{2}}, y^{s_{1}}\right)$ from Proposition $6, H(x, y)$ has a real factor $a x^{s_{2}}+b y^{s_{1}}$. If one of $a$ and $b$ is zero, then $H(x, y)$ has a real linear factor, which contradicts to the fact $H(x, y)$ has no real linear factors. Hence, the conclusion (c) holds. Next we consider the case $a b \neq 0$. It can be checked that $a x^{s_{2}}+b y^{s_{1}}$ is a Darboux polynomial of system (2). Hence, $a x^{s_{2}}+b y^{s_{1}}=0$ is invariant for system (2). Note that $s_{1}$ and $s_{2}$ are coprime by Proposition ??. Thus, one of $s_{1}$ and $s_{2}$ is odd. Without loss of generality, we assume that $s_{1}$ is odd. Then the curve $y=\left(-\frac{a}{b}\right)^{\frac{1}{s_{1}}} x^{\frac{s_{2}}{s_{1}}}$ is an invariant curve of system (2), which passes through the origin $O(0,0)$. This contradicts with the fact that $O(0,0)$ is center.

Hence, the "if" part of the theorem is proved.

Now we prove the "only if" part. If the condition (c) holds, that is, the homogeneous polynomial $H^{*}(x, y)$ has no real linear factors, then 
the degree $\frac{m}{s_{1} s_{2}}$ of $H^{*}(x, y)$ must be even denoted by $2 l, H^{*}(1, z)=0$ has no real roots and $H^{*}(0,1) \neq 0$. Without loss of generality, we assume that $H^{*}(0,1)>0$. Then $H^{*}(1, z)>0$, otherwise, $H^{*}(x, y)$ has real linear factors.

Therefore, $H^{*}(0, y)=H^{*}(0,1) y^{2 l}>0$ for any $y \neq 0$, and $H^{*}(x, y)=$ $H^{*}(1, z) x^{2 l}>0$ for any $x \neq 0$. This implies that

$$
H^{*}(x, y)>H^{*}(0,0)=0,
$$

that is, $O(0,0)$ is the extreme minimum point of $H^{*}(x, y)$ in $\mathbb{R}^{2}$.

Note that $H^{*}(x, y)=H\left(x^{\frac{1}{s_{2}}}, y^{\frac{1}{s_{1}}}\right)$. Hence, $H(x, y)=H^{*}\left(x^{s_{2}}, y^{s_{1}}\right)$. It can be easily checked that $O(0,0)$ is the extreme minimum point of $H(x, y)$ in $\mathbb{R}^{2}$. Thus, system (2) has a first integral $H(x, y)$ with an isolated minimum at $O(0,0)$, which implies that $O(0,0)$ is a center of system (2). We complete the proof of the "only if" part.

Finally we shall prove that the center is always global.

Following the method in [14], we consider the Liapunov $\left(s_{1}, s_{2}\right)$ trigometric functions introduced by [11].

$$
x=r^{s_{1}} C s \theta, \quad y=r^{s_{2}} \operatorname{Sn} \theta,
$$

where $C \sin$ and $\operatorname{Sn} \theta$ are periodic functions with period $\tau$, where $\tau=$ $2 s_{1}^{-\frac{1}{2 s_{2}}} s_{2}^{-\frac{1}{2 s_{1}}} B\left(\frac{1}{2 s_{1}}, \frac{1}{2 s_{2}}\right)$ and $B$ is the $\beta$ function

$$
B(\alpha, \beta)=2 \int_{0}^{\frac{\pi}{2}} \sin ^{2 \alpha-1} \theta \cos ^{2 \beta-1} \theta d \theta .
$$

And

$$
r^{2 s_{1} s_{2}}=s_{1} x^{2 s_{2}}+s_{2} y^{2 s_{1}}, \quad \frac{S n^{s_{1}} \theta}{C s^{s_{2}} \theta}=\frac{y^{s_{1}}}{x^{s_{2}}} .
$$

This transformation (9) is bi-analytic from $(0,+\infty) \times \mathbb{S}^{1}$ to $\mathbb{R}^{2} \backslash\{0\}$, which transforms system (2) to

$$
\begin{aligned}
\dot{r} & =-r^{m-s_{1}-s_{2}+1}\left(C s^{2 s_{2}-1} \theta \frac{\partial H}{\partial y}(C s \theta, S n \theta)-S^{2 s_{1}-1} \theta \frac{\partial H}{\partial x}(C s \theta, S n \theta)\right), \\
\dot{\theta} & =r^{m-s_{1}-s_{2}}\left(s_{1} C s \theta \frac{\partial H}{\partial x}(C s \theta, \operatorname{Sn} \theta)+s_{2} \operatorname{Sn} \theta \frac{\partial H}{\partial y}(C s \theta, \operatorname{Sn} \theta)\right) .
\end{aligned}
$$

Note that $H(x, y)$ is quasi-homogenous. Thus,

$$
s_{1} C s \theta \frac{\partial H}{\partial x}(C s \theta, \operatorname{Sn} \theta)+s_{2} \operatorname{Sn} \theta \frac{\partial H}{\partial y}(C \sin , \operatorname{Sn} \theta)=m H(C \sin , \operatorname{Sn} \theta)
$$


which is either positive, or negative, for all $\theta$ because $H(x, y)$ has no real linear factors. Therefore, system (10) can be written as

$$
\frac{d r}{d \theta}=-r \frac{C s^{2 s_{2}-1} \theta \frac{\partial H}{\partial y}(C s \theta, \operatorname{Sn} \theta)-S^{2 s_{1}-1} \theta \frac{\partial H}{\partial x}(C s \theta, \operatorname{Sn} \theta)}{s_{1} C s \theta \frac{\partial H}{\partial x}(C s \theta, \operatorname{Sn} \theta)+s_{2} \operatorname{Sn} \theta \frac{\partial H}{\partial y}(C s \theta, \operatorname{Sn} \theta)} .
$$

Let

$$
F(\theta)=\frac{C s^{2 s_{2}-1} \theta \frac{\partial H}{\partial y}(C s \theta, \operatorname{Sn} \theta)-S^{2 s_{1}-1} \theta \frac{\partial H}{\partial x}(C s \theta, \operatorname{Sn} \theta)}{s_{1} C s \theta \frac{\partial H}{\partial x}(C s \theta, S n \theta)+s_{2} \operatorname{Sn} \theta \frac{\partial H}{\partial y}(C s \theta, S n \theta)} .
$$

The system (12) has a solution with the initial condition $\left(0, r_{0}\right)$ for any $r_{0}$ as follows

$$
r\left(\theta, 0, r_{0}\right)=r_{0} e^{-\int_{0}^{\theta} F(s) d s} .
$$

If $O(0,0)$ is a center, then for any given real number $0<h \ll 1$, the level set $H(x, y)=h$, that is, $H\left(r^{s_{1}} C s \theta, r^{s_{2}} \operatorname{Sn} \theta\right)=r^{m} H(C s \theta, \operatorname{Sn} \theta)=$ $h$, is a closed orbit. Thus, $r_{0}=\left(\frac{h}{H(C s 0, S n 0)}\right)^{\frac{1}{m}}$ and $r\left(2 \pi, 0, r_{0}\right)=r_{0}$. This implies that the integral $\int_{0}^{\tau} F(s) d s \equiv 0$. Thus, for any real number $h>0$, the orbit $r\left(\theta, 0,\left(\frac{h}{H(C s 0, S n 0)}\right)^{\frac{1}{m}}\right)=\left(\frac{h}{H(C s 0, S n 0)}\right)^{\frac{1}{m}}$ of system $(12)$ is closed. This leads that the center $O(0,0)$ is global in $\mathbb{R}^{2}$.

\section{A small perturbation of homogeneous Hamiltonian SYSTEMS WITH CENTER}

Consider system (1) with homogeneous polynomial Hamiltonian $H(x, y)$ with degree $m, m \in \mathbb{N}$. We calculate the higher-order Melnikov functions $M_{k}(h)$ of (1) and estimate an upper bound for the number of their isolated zeros in the interval $\left(0, h_{1}\right)$. Let $\omega$ be a polynomial differential 1 -form

$$
\omega=p(x, y) d x+q(x, y) d y,
$$

where $p(x, y)$ and $q(x, y)$ are real polynomials with degree at most $n$, $n \in \mathbb{N}$. Then system (1) can be written as

$$
d H+\epsilon \omega=0
$$

where $\epsilon$ is a small parameter and $H=H(x, y)$. 
Denote $C=x \frac{\partial}{\partial x}+y \frac{\partial}{\partial y}$ the Euler vector field. The fact that $H$ is homogeneous of degree $m$ is equivalent to

$$
C . H=x \frac{\partial H}{\partial x}+y \frac{\partial H}{\partial y}=m H .
$$

We follow the method introduced in [2] and improved in [6] which is based on iterated path integral and the Gelfand-Leray derivative (see also [3]).

Let furthermore introduce the polynomial $D_{1}(x, y)$ so that:

$$
d \omega=D_{1}(x, y) d x \wedge d y,
$$

and the Gelfand-Leray derivative of $\omega$ :

$$
\omega_{1}=\frac{1}{m H} D_{1}(x, y) \iota_{C} d x \wedge d y:=\frac{d \omega}{d H},
$$

where $\iota_{C}$ means the interior product by the Euler vector field $C$. This displays the relation:

$$
d \omega=\omega_{1} \wedge d H
$$

Conversely, if $H$ has an isolated critical point at the origin $O(0,0)$, then the above relation characterizes the Gelfand-Leray 1-form up to a multiple of $d H$. Assume that $H$ defines a regular fibration $H$ : $U \rightarrow\left(0, h_{1}\right)$, where $U$ is an annular-shaped open set on the plane $\mathbb{R}^{2}$ and $\left(0, h_{1}\right)$ is an open interval of the real line $\mathbb{R}$. For $h \in\left(0, h_{1}\right)$, $\gamma_{h}=H^{-1}(h) \cap U$ is an oval. Let $\sigma$ be a transverse section of the flow of $(14)$, for example, taking an open interval $\left(0, h_{1}\right)$ in the $x$-axis. Given a 1 -form $\omega$, define the multivalued function $f$ on the complement of the section $\sigma$ along the flow of Hamiltonian system (2), $f: U \backslash \sigma \subset \mathbb{R}^{2} \rightarrow$ $\mathbb{R}, M \in U \backslash \sigma \mapsto f(M)$ :

$$
f(M)=\int_{\gamma_{S_{0}(h) M}} \omega,
$$

where $\gamma_{S_{0}(h) M}$ is an orbit of $(2)$ between the point $S_{0}(h)$ and the point $M, S_{0}(h)=\gamma_{h} \cap \sigma$. The integral of 1 -form $\omega$ is taken along $\gamma_{\widehat{S_{0}(h) M}}$. A shorter notation for (19) can be conveniently used as:

$$
f=\int_{S_{0}(h)}^{(x, y)} \omega,
$$

where $(x, y)$ denotes the coordinates of the point $M$ in $U \backslash \sigma \subset \mathbb{R}^{2}$. The following lemma was proved in [3]: 
Lemma 7. Any analytic 1-form $\omega$ can be written in the form:

$$
\omega=g_{1} d H+d f_{1},
$$

with multi-valued analytic functions $f_{1}$ and $g_{1}$ defined outside of the cross-section $\sigma$ as follows:

$$
f_{1}=\int_{S_{0}(h)}^{(x, y)} \omega, g_{1}=\int_{S_{0}(h)}^{(x, y)} \omega_{1}+R_{1}(H),
$$

where $R_{1}(H)$ is a function of $H$ alone.

Proof. By the definition of the Gelfand-Leray form $\omega_{1}$, we obtain that:

$$
d \omega=d\left(\int_{S_{0}(h)}^{(x, y)} \omega_{1}\right) \wedge d H .
$$

Hence there exists a function $f_{1}$ such that:

$$
\omega=\left(\int_{S_{0}(h)}^{(x, y)} \omega_{1}\right) d H+d f_{1} .
$$

Integrating from $S_{0}(h)$ to the point $(x, y)$ along the level set $\gamma_{h}=$ $\{(x, y): H(x, y)=h\}$ of $H$ yields:

$$
\int_{S_{0}(h)}^{(x, y)} \omega=f_{1}(x, y)-f_{1}\left(S_{0}(h)\right) .
$$

The function $f_{1}\left(S_{0}(h)\right)$ is constant along the level sets of $H$ and therefore is a function of $H$ alone. Then we can choose $f_{1}=\int_{S_{0}(h)}^{(x, y)} \omega$ and write

$$
\omega=\left[\int_{S_{0}(h)}^{(x, y)} \omega_{1}+R_{1}(H)\right] d H+d\left(\int_{S_{0}(h)}^{(x, y)} \omega\right) .
$$

In [2], the algorithm of the successive derivatives was introduced to obtain the first non-zero Melnikov function if the Hamiltonian satisfies the $*$-property relatively to a fixed period annulus $U$. The $*$-property is as follow. 
Definition 8. For any polynomial 1-form $\omega$ such that

$$
\oint_{\gamma_{h}} \omega=0
$$

for all $h \in H(U), H(U)$ is an interval in $\mathbb{R}$, then there exist analytic functions $g$ and $R$ on $U$, such that

$$
\omega=g d H+d R
$$

In [2], it was shown that $H=\frac{1}{2}\left(x^{2}+y^{2}\right)$ satisfies the $*$-property, with polynomials $g$ and $R$. An example of Hamiltonian system is given in [13] to show that the $*$-property does not always hold with polynomials, where Hamiltonian function is

$$
H(x, y)=\frac{y^{2}}{2}+\frac{\left(x^{2}-1\right)^{2}}{4}
$$

There is a 1-form $\eta=x y d x$ such that $\oint_{\gamma_{h}} \eta=0$ for $h>\frac{1}{4}$, but $\oint_{\delta_{ \pm}(h)} \eta \neq 0$, where $\delta_{ \pm}(h)$ is one $\gamma_{h}$ 's component around singularity $(x, y)=( \pm 1,0)$ for $0<h<\frac{1}{4}$, respectively (for details refer to $[12,13]$ ). In some sense, the lemma 8 tells that the $*$-property is always satisfied for analytic functions on the complement of the cross-section (instead of polynomials). It is remarkable that this is sufficient in general to bound the number of zeroes of the first non-zero Melnikov function (also called the bifurcation function in [4]).

3.1. The first Melnikov function. It is well known that the first Melnikov function of (14) is $M_{1}(h)=\oint_{\gamma_{h}} \omega$, where

$$
\omega=\Sigma_{0 \leq i+j \leq n} \lambda_{i j} x^{i} y^{j} d x+\Sigma_{0 \leq i+j \leq n} \mu_{i j} x^{i} y^{j} d y
$$

where $\lambda_{i j}$ and $\mu_{i j}$ are the functions of coefficients of polynomial perturbations $p(x, y)$ and $q(x, y)$.

Let consider the change of variables $\phi:(x, y) \mapsto\left(h^{\frac{1}{m}} x, h^{\frac{1}{m}} y\right)$ and denote by $\phi *$ the corresponding image. Then, we obtain

$$
\begin{aligned}
\phi^{*}(\omega) & =\sum_{k=0}^{n}\left\{\Sigma_{i+j=k}\left[\lambda_{i j} x^{i} y^{j} d x+\mu_{i j} x^{i} y^{j} d y\right] h^{\frac{k+1}{m}}\right\}, \\
\phi^{*}\left(\gamma_{h}\right) & =\gamma_{1} .
\end{aligned}
$$


This yields

$$
\begin{aligned}
M_{1}(h) & =\oint_{\gamma_{h}} \omega=\oint_{\gamma_{1}} \phi^{*}(\omega) \\
& =\oint_{\gamma_{1}} \sum_{k=0}^{n}\left\{\sum_{i+j=k}\left[\lambda_{i j} x^{i} y^{j} d x+\mu_{i j} x^{i} y^{j} d y\right] h^{\frac{k+1}{m}}\right\} \\
& =\sum_{k=0}^{n} \alpha_{k}(\lambda, \mu) h^{\frac{k+1}{m}}
\end{aligned}
$$

where

$$
\alpha_{k}(\lambda, \mu)=\oint_{\gamma_{1}} \Sigma_{i+j=k}\left[\lambda_{i j} x^{i} y^{j} d x+\mu_{i j} x^{i} y^{j} d y\right],
$$

which are linear functions of the parameters $\lambda_{i j}, \mu_{i j}$ of the perturbation. It is then easy to deduce:

Lemma 9. The number of isolated real zeros of the first Melnikov function $M_{1}(h)$ is less or equal than $n+1$.

Remark 10. This is actually not optimal in general because in our context, we are only concerned with the number of isolated and positive zeros of $M_{1}(h)$.

3.2. The higher-order Melnikov functions. In this part, we show inductively on the higher-order Melnikov functions $M_{k}$ for the integer $k$, $k \geq 2$ if $M_{1}(h) \equiv 0$. It shows that the bifurcation function $h^{k-1} M_{k}(h)$ is a polynomial function in $h^{\frac{1}{m}}$. We begin with some lemmas.

An analytical 1-form $\omega$ is called homogeneous 1-form of degree $n$ if $\omega=P(x, y) d x+Q(x, y) d y$ and $P(x, y), Q(x, y)$ are homogenous functions of degree $n$. And an analytical 1-form $\omega$ is called 1-form of degree $n$ (at most $n$ ) if the highest homogenous degree of homogenous component of $\omega$ is $n$ (at most $n$ ).

Lemma 11. If $\omega$ is a homogeneous 1-form of degree $n$ and $H(x, y)$ is homogeneous of degree $m$, then the integral of $\omega$ along $\gamma_{h}$ of Hamiltonian system $(2)$ from $S_{0}(h)$ to point $(x, y), F(x, y)=\int_{S_{0}(h)}^{(x, y)} \omega$, is a homogenous function of degree $n+1$.

Proof. Choosing a transverse section $\sigma$ on a line passing by the origin, we consider the level set $\gamma_{h}$ of system (2) and let $S_{0}(h)=\gamma_{h} \cap \sigma$. Then $S_{0}\left(\lambda^{m} h\right)=\lambda S_{0}(h)$ and $H(\lambda x, \lambda y)=\lambda^{m} H(x, y)$ since $H(x, y)$ is homogeneous of degree $m$. Hence,

$$
F(\lambda x, \lambda y)=\int_{S_{0}\left(\lambda^{m} h\right)}^{(\lambda x, \lambda y)} \omega .
$$


Note that $\omega=P(x, y) d x+Q(x, y) d y$, and $P(x, y)$ and $Q(x, y)$ are homogenous functions of degree $n$. Let $x=\lambda X$ and $y=\lambda Y$. Then this yields

$$
\begin{aligned}
F(\lambda x, \lambda y) & =\int_{\lambda S_{0}(h)}^{(\lambda x, \lambda y)} P(x, y) d x+Q(x, y) d y \\
& =\int_{S_{0}(h)}^{(x, y)} \lambda(P(\lambda X, \lambda Y) d X+Q(\lambda X, \lambda Y) d Y) \\
& =\lambda^{n+1} \int_{S_{0}(h)}^{(x, y)}(P(X, Y) d X+Q(X, Y) d Y) \\
& =\lambda^{n+1} F(x, y) .
\end{aligned}
$$

That means $F(x, y)$ is a homogenous function of degree $n+1$. The proof is finished.

Lemma 12. If the first Melnikov function $M_{1}(h)$ is identically zero, then the number of zeroes of $M_{2}(h)$ is at most $2(n+1)$. More precisely, $h M_{2}(h)$ is polynomial in $h^{\frac{1}{m}}$ of degree at most $2(n+1)$.

Proof. If $M_{1}(h) \equiv 0$, the higher order Melnikov function $M_{2}(h)$ should be considered. To compute $M_{2}(h)$, we have first to consider $\omega_{1}=$ $\frac{1}{m H} D_{1}(x, y) \iota_{C} d x \wedge d y$. Denote $\bar{\omega}_{1}=\frac{1}{m} D_{1}(x, y) \iota_{C} d x \wedge d y$. The function $M_{2}(h)$ is given by:

$$
M_{2}(h)=\frac{1}{h} \oint_{\gamma_{h}}\left[\int_{S_{0}(h)}^{(x, y)} \bar{\omega}_{1}\right] \omega .
$$

We proceed with the change of variables $\phi:(x, y) \mapsto\left(h^{\frac{1}{m}} x, h^{\frac{1}{m}} y\right)$ in the integral:

$$
M_{2}(h)=\frac{1}{h} \oint_{\gamma_{1}} \phi^{*}\left[\int_{S_{0}(h)}^{(x, y)} \bar{\omega}_{1}\right] \phi^{*} \omega .
$$

The equation (31) shows that the 1 -form $\phi^{*} \omega$ is polynomial in $h^{\frac{1}{m}}$ of degree at most $n+1$. Note that $\bar{\omega}_{1}$ is a 1 -form of degree at most $n$. The lemma 11 shows that the 1 -form $\phi^{*}\left[\int_{S_{0}(h)}^{(x, y)} \bar{\omega}_{1}\right]$ is then also polynomial in $h^{\frac{1}{m}}$ of degree at most $n+1$. It is then clear that the product of $\phi^{*}\left[\int_{S_{0}(h)}^{(x, y)} \bar{\omega}_{1}\right]$ and $\phi^{*} \omega$ is polynomial in $h^{\frac{1}{m}}$ of degree at most $2(n+1)$. This yields that the function $h M_{2}(h)$ is polynomial in $h^{\frac{1}{m}}$ of degree at most $2(n+1)$ and has at most $2(n+1)$ zeroes. 
It is now ready to prove the following theorem.

Theorem 13. The bifurcation function $M_{k}(h)$ of (14) displays at most $k(n+1)$ isolated zeros for any natural number $k$.

Proof. The conclusion is true if $k=1$ and $k=2$ by Lemma 11 and Lemma 12. Using the induction, we assume that up to order $k$,

$$
M_{k}(h)=\frac{1}{h^{k-1}} \oint_{\gamma_{h}} \bar{g}_{k-1} \omega
$$

where $\bar{g}_{k-1}(x, y)=\int_{S_{0}(h)}^{(x, y)} \bar{\omega}_{k-1}$ and $\bar{\omega}_{k-1}$ is 1 -form of degree at most $(n+1)(k-1)-1$. Then by lemma $11, \bar{g}_{k-1}$ is a function of degree at most $(n+1)(k-1)$.

Let $g_{k-1}=\frac{\bar{g}_{k-1}}{H^{k-1}}$ and $\omega_{k-1}=g_{k-1} \omega$. Then assume that $M_{k}(h) \equiv 0$, the algorithm of the successive derivatives implies that there exist $g_{k}$ and $R_{k}$ such that

$$
g_{k-1} \omega=g_{k} d H+d R_{k} .
$$

The function $g_{k}$ is given by

$$
g_{k}(x, y)=\int_{S_{0}(h)}^{(x, y)} \omega_{k},
$$

where

$$
\begin{aligned}
\omega_{k} & =\frac{1}{m H} \iota_{C} d\left(g_{k-1} \omega\right)=\frac{1}{m H} \iota_{C} d\left(\frac{\bar{g}_{k-1}}{H^{k-1}} \omega\right) \\
& =\frac{1}{m H^{k}} \iota_{C} d\left(\bar{g}_{k-1} \omega\right)-\frac{k-1}{m H} \iota_{C}\left(\bar{g}_{k-1} \frac{d H}{H^{k}} \wedge \omega\right) .
\end{aligned}
$$

This yields

$$
\begin{aligned}
g_{k} & =\int_{S_{0}(h)}^{(x, y)} \frac{1}{H^{k}}\left[\frac{1}{m} \iota_{C} d\left(\bar{g}_{k-1} \omega\right)-(k-1) \bar{g}_{k-1} \omega\right] \\
& =\frac{1}{H^{k}} \int_{S_{0}(h)}^{(x, y)} \bar{\omega}_{k},
\end{aligned}
$$

where $\bar{\omega}_{k}=\frac{1}{m} \iota_{C} d\left(\bar{g}_{k-1} \omega\right)-(k-1) \bar{g}_{k-1} \omega$, which is a 1-form of degree at most $(n+1) k-1$. Hence,

$$
M_{k+1}(h)=\frac{1}{h^{k}} \oint_{\gamma_{h}} \bar{g}_{k} \omega .
$$

It is then obvious that $h^{k} M_{k+1}(h)$ is a polynomial in $h^{\frac{1}{m}}$ of degree at most $(n+1)(k+1)$, and the theorem is proved. 


\section{A small PERTURBation OF QUASI-HomogeneOUS HAMILTONIAN SYSTEMS WITH (GLOBAL) CENTER}

In this section, we consider the number of limit cycles bifurcating from the finite plane $\mathbb{R}^{2}$ for system (1) in which Hamiltonian function $H(x, y)$ is an $\left(s_{1}, s_{2}\right)$-quasi-homogeneous polynomial of weight degree $m$, and $p(x, y)$ and $q(x, y)$ are real polynomials of degree at most $n$. Theorem 2 tells when this quasi-homogeneous polynomial Hamiltonian system (2) has a center. When system (2) has a center which is global, we study the first nonzero $k$ th order Melnikov functions $M_{k}(h)$ of system (1). Without loss of generality, let $s_{1} \geq s_{2}>0$ for quasihomogeneous polynomial $H(x, y)$. For any given $s_{1}$ and $s_{2}$, the perturbation polynomials $p(x, y)$ and $q(x, y)$ of (1) can be decomposed into the sum of the $\left(s_{1}, s_{2}\right)$-quasi-homogeneous polynomials of weight degree $i$, that is,

$$
\begin{aligned}
& p\left(\lambda^{s_{1}} x, \lambda^{s_{2}} y\right)=\sum_{i=0}^{n s_{1}} p_{i}(x, y) \lambda^{i}, \\
& q\left(\lambda^{s_{1}} x, \lambda^{s_{2}} y\right)=\sum_{i=0}^{n s_{1}} q_{i}(x, y) \lambda^{i},
\end{aligned}
$$

where $p_{i}(x, y)$ and $q_{i}(x, y)$ are $\left(s_{1}, s_{2}\right)$-quasi-homogeneous polynomials of weight degree $i$.

And this decomposition is obviously unique with respective to $s_{1}$ and $s_{2}$. Especially, when $s_{1}=s_{2}=1,(37)$ is a homogeneous decomposition. Therefore, the polynomial differential 1-form (13) becomes

$$
\omega=\sum_{i=0}^{n s_{1}} w_{i}, w_{i}=p_{i}(x, y) d x+q_{i}(x, y) d y
$$

The main result in this section is as follows:

Theorem 14. Assume that $H(x, y)$ is an $\left(s_{1}, s_{2}\right)$-quasi-homogeneous polynomial of weight degree $m$, and $p(x, y)$ and $q(x, y)$ are polynomials of degree at most $n$. If Hamiltonian system (2) has a global center at $O(0,0)$, then 
(I) the first nonzero $k$ th order Melnikov functions $M_{k}(h)$ of system (1) can be expressed as

$$
M_{k}(h)=\sum_{i=\left(s_{2}-m\right)(k-1)+s_{2}}^{\left((n+1) s_{1}-m\right)(k-1)+(n+1) s_{1}} a_{i} h^{\frac{i}{m}}, \quad 0<h<+\infty,
$$

where $a_{i}$ is constant and $a_{i}=0$ if both $i$ is even and $\left(s_{1}+s_{2}\right)$ is odd; or all $i, s_{1}$ and $s_{2}$ are odd.

(II) $M_{k}(h)$ have at most $K$ zeros on $(0,+\infty)$, where

$$
K= \begin{cases}\frac{k\left((n+1) s_{1}-s_{2}\right)}{2}-1, & k \text { is even, and all } k, s_{1}, s_{2} \text { are odd; } \\ & s_{2} \text { is even, and } s_{1}+s_{2} \text { is odd } k, s_{1}, n \text { is odd } \\ \frac{k\left((n+1) s_{1}-s_{2}\right)}{2}, & k \text { is even, and both } s_{1} \text { and } s_{2} \text { are odd; } \\ \frac{k\left((n+1) s_{1}-s_{2}\right)-1}{2}, & \text { others. }\end{cases}
$$

When $s_{1}=s_{2}=1$, the quasi-homogeneous polynomial $H(x, y)$ becomes homogeneous polynomial of degree $m$. Then Theorem $14 \mathrm{im}-$ proves Theorem 13 in section 2 . Hence, we directly obtain the following conclusion by Corollary 3 and Theorem 14 .

Theorem 15. Assume that $H(x, y)$ is a homogeneous polynomial of degree $m$ and $H(x, y)$ has no real linear factors. Then

(A) the bifurcation function $M_{k}(h)$ of system (1) can be expressed as

$$
M_{k}(h)= \begin{cases}h^{\frac{(1-m)(k-1)+2}{m}} \sum_{i=0}^{\left[\frac{k n-1}{2}\right]} \tilde{a}_{i}\left(h^{\frac{2}{m}}\right)^{i}, & k \text { is odd }, \\ h^{\frac{(1-m)(k-1)+1}{m}} \sum_{i=0}^{\frac{k n}{2}} \tilde{a}_{i}\left(h^{\frac{2}{m}}\right)^{i}, & k \text { is even },\end{cases}
$$

where $\tilde{a}_{i}$ is constant.

(B) $M_{k}(h)$ has at most $\left[\frac{k n-1}{2}\right]$ zeros on $(0,+\infty)$ if $k$ is odd, and $M_{k}(h)$ has at most $\frac{k n}{2}$ zeros on $(0,+\infty)$ if $k$ is even, where $[x]$ means the maximum integer not more than $x$.

Theorem 15 extends the results of Iliev in [9] and Buica et al. in [1].

To prove Theorem 14, we first state some propositions and lemmas. The following proposition is easily checked. 
Proposition 16. Assume that $F(x, y)$ is an $\left(s_{1}, s_{2}\right)$-quasi-homogeneous function of weight degree $m$. Then

(i) $\frac{\partial F}{\partial x}\left(\frac{\partial F}{\partial y}\right)$ is an $\left(s_{1}, s_{2}\right)$-quasi-homogeneous function of weight degree $m-s_{1}\left(m-s_{2}\right.$, resp.);

(ii) $x F(y F)$ is an $\left(s_{1}, s_{2}\right)$-quasi-homogeneous function of weight degree $m+s_{1}\left(m+s_{2}\right.$, resp. $)$;

(iii) $\int_{S_{0}(h)}^{(x, y)} F d x\left(\int_{S_{0}(h)}^{(x, y)} F d y\right)$ is an $\left(s_{1}, s_{2}\right)$-quasi-homogeneous function of weight degree $m+s_{1}\left(m+s_{2}\right.$, resp.).

Lemma 17. If $M_{k}(h)=\oint_{\gamma_{h}} \Omega_{k}$, then

$$
\Omega_{k}=\sum_{i=\left(s_{2}-m\right)(k-1)}^{\left((n+1) s_{1}-m\right)(k-1)+n s_{1}} \Omega_{k, i}
$$

where $\Omega_{k, i}$ is the $\left(s_{1}, s_{2}\right)$-quasi-homogeneous items with weight degree $i$ of $\Omega_{k}$.

Proof. Using the induction, we consider $k=1$,

$$
\Omega_{1}=\omega=\sum_{i=0}^{n s_{1}} \Omega_{1, i}
$$

where $\Omega_{1, i}=w_{i}, w_{i}$ is the $\left(s_{1}, s_{2}\right)$-quasi-homogenous item with weight degree $i$ of $\omega$ by (38).

Assume that (39) holds when $k=l$. We claim that (39) holds for $k=l+1$.

Indeed, let $\Delta \Omega_{l}$ be the divergence of $\Omega_{l}$ and let

$$
\eta=\frac{1}{m H} \Delta \Omega_{l} \iota_{C} d x \wedge d y=\frac{\Delta \Omega_{l}\left(s_{2} y d x-s_{1} x d y\right)}{m H} .
$$

Then

$$
d \Omega_{l}=\Delta \Omega_{l} d x \wedge d y=\eta \wedge d H .
$$

By proposition 16, the lowest weight degree of quasi-homogenous item of $\Delta \Omega_{l}$ is $\left(s_{2}-m\right)(l-1)-s_{1}$ and the highest weight degree is $((n+$ 1) $\left.s_{1}-m\right)(l-1)+n s_{1}-s_{2}$. Thus,

$$
\eta=\sum_{i=\left(s_{2}-m\right)(l-1)-s_{1}}^{\left((n+1) s_{1}-m\right)(l-1)+n s_{1}-s_{2}} \eta_{1, i} d x+\sum_{i=\left(s_{2}-m\right)(l-1)-s_{1}}^{\left((n+1) s_{1}-m\right)(l-1)+n s_{1}-s_{2}} \eta_{2, i} d y
$$

where $\eta_{1, i}=\frac{s_{2}\left(\Delta \Omega_{l}\right)_{i} y}{m H}$ is the quasi-homogenous with weight degree $i+$ $s_{2}-m, \eta_{2, i}=-\frac{s_{1}\left(\Delta \Omega_{l}\right)_{i} x}{m H}$ is the quasi-homogenous with weight degree 
$i+s_{1}-m$, and $\left(\Delta \Omega_{l}\right)_{i}$ is the quasi-homogenous items with weight degree $i$ of $\Delta \Omega_{l}$. So we have

$$
\Omega_{l+1}=\omega \int_{S_{0}(h)}^{(x, y)} \eta=\sum_{i=\left(s_{2}-m\right)(l-1)-s_{1}}^{\left((n+1) s_{1}-m\right)(l-1)+n s_{1}-s_{2}} \sum_{j=0}^{n s_{1}} w_{j} \int_{S_{0}(h)}^{(x, y)} \eta_{1, i} d x+\eta_{2, i} d y,
$$

By proposition $16, \int_{S_{0}(h)}^{(x, y)} \eta_{1, i} d x+\eta_{2, i} d y$ is an $\left(s_{1}, s_{2}\right)$-quasi-homogenous function of weight degree $i+s_{1}+s_{2}-m$.

So the items of $\Omega_{l+1}$ with lowest weight degree are

$$
w_{0} \int_{S_{0}(h)}^{(x, y)} \eta_{1,\left(s_{2}-m\right)(l-1)-s_{1}} d x+\eta_{2,\left(s_{2}-m\right)(l-1)-s_{1}} d y
$$

and its weight degree is $\left(s_{2}-m\right)(l-1)-s_{1}+s_{1}+s_{2}-m=\left(s_{2}-m\right) l$. The items of $\Omega_{l+1}$ with the highest weight degree are

$$
w_{n s_{1}} \int_{S_{0}(h)}^{(x, y)} \eta_{1,\left((n+1) s_{1}-m\right)(l-1)+n s_{1}-s_{2}} d x+\eta_{2,\left((n+1) s_{1}-m\right)(l-1)+n s_{1}-s_{2}} d y,
$$

whose weight degree is $n s_{1}+\left((n+1) s_{1}-m\right)(l-1)+n s_{1}-s_{2}+s_{1}+s_{2}-m=\left((n+1) s_{1}-m\right) l+n s_{1}$. This leads that (39) holds for $k=l+1$.

Now we are in the position to prove Theorem 14.

Proof of Theorem 14. By Lemma 17, using the transformation of variables

we have

$$
(x, y) \mapsto\left(h^{\frac{s_{1}}{m}} x, h^{\frac{s_{2}}{m}} y\right),
$$

$$
M_{k}(h)=\sum_{i=\left(s_{2}-m\right)(k-1)+s_{2}}^{\left((n+1) s_{1}-m\right)(k-1)+(n+1) s_{1}} a_{i} h^{\frac{i}{m}}, 0<h<+\infty .
$$

where

$$
a_{i}=\left\{\begin{array}{l}
\oint_{\gamma_{1}} \Omega_{2, k, i-s_{2}} d y, \quad i<L_{1}, \\
\oint_{\gamma_{1}} \Omega_{1, k, i-s_{1}} d x+\oint_{\gamma_{1}} \Omega_{2, k, i-s_{2}} d y, \quad L_{1} \leq i \leq L_{2}, \\
\oint_{\gamma_{1}} \Omega_{1, k, i-s_{1}} d x, \quad i>L_{2},
\end{array}\right.
$$

here $L_{1}=\left(s_{2}-m\right)(k-1)+s_{1}, L_{2}=\left((n+1) s_{1}-m\right)(k-1)+n s_{1}+s_{2}$, and $\Omega_{k, i}=\Omega_{1, k, i} d x+\Omega_{2, k, i} d y$. Thus, $a_{i}$ is constant for any $i$. 
Furthermore, we claim that $a_{i}=0$ as both $i$ is even and $\left(s_{1}+s_{2}\right)$ is odd; or as all $i, s_{1}$ and $s_{2}$ are odd.

Indeed, by theorem $2, m$ must be even and there are two cases:

(1) $s_{1}+s_{2}$ is odd;

(2) $s_{1}$ is odd and $s_{2}$ is odd.

If the case (1) holds, that is, $s_{1}+s_{2}$ is odd, then let

$$
x \mapsto(-1)^{s_{1}} x, y \mapsto(-1)^{s_{2}} y,
$$

and

$$
\begin{aligned}
& \oint_{\gamma_{1}} \Omega_{1, k, i-s_{2}} d y=(-1)^{1+i} \oint_{\gamma_{1}} \Omega_{1, k, i-s_{2}} d y, \\
& \oint_{\gamma_{1}} \Omega_{2, k, i-s_{1}} d x=(-1)^{1+i} \oint_{\gamma_{1}} \Omega_{2, k, i-s_{1}} d x .
\end{aligned}
$$

So we have $a_{i}=-a_{i}$ if $i$ is even. Hence, $a_{i}=0$. This implies that $a_{i}=0$ if $i$ is even and $s_{1}+s_{2}$ is odd. Similar arguments to the case (2) shows that $a_{i}=0$ if $i$ is odd, and both $s_{1}$ and $s_{2}$ are odd.

By expression $M_{k}(h)$ in conclusion (I), it follows that $M_{k}(h)$ have at most $K$ zeros on $(0,+\infty)$, where

$$
K= \begin{cases}\frac{k\left((n+1) s_{1}-s_{2}\right)}{2}-1, & k \text { is even, and all } k, s_{1}, s_{2} \text { are odd } \\ & s_{2} \text { is even, and } s_{1}+s_{2} \text { is odd } \\ \frac{k\left((n+1) s_{1}-s_{2}\right)}{2}, & k \text { is even, } s_{1}, s_{2} \text { are odd }, \\ \frac{k\left((n+1) s_{1}-s_{2}\right)-1}{2}, & \text { others. }\end{cases}
$$

Remark The upper bound $K$ for isolated zeros of the $k$-order Melnikov function $M_{k}(h)$ in Theorem 14 usually is not the optimal one. Let us see the following examples.

Example: consider system

$$
\begin{aligned}
& \frac{d x(t)}{d t}=-4 y^{3}+\epsilon \sum_{0 \leq i+j \leq 2} a_{i j} x^{i} y^{j}, \\
& \frac{d y(t)}{d t}=4 x+\sum_{0 \leq i+j \leq 2} b_{i j} x^{i} y^{j},
\end{aligned}
$$

where $|\epsilon|<<1, a_{i j}$ and $b_{i j}$ are real parameters. Then 
(i) $M_{1}(h)$ has no zeros on $(0,+\infty)$;

(ii) $M_{2}(h)$ has at most 3 zeros on $(0,+\infty)$ if $M_{1}(h) \equiv 0$. And the three is the optimal upper bound of $M_{2}(h)$;

(iii) $M_{3}(h)$ has at most 2 zeros on $(0,+\infty)$ if $M_{1}(h)=M_{2}(h) \equiv 0$. And the two zeros of $M_{3}(h)$ can be reached.

It is clear that the actual upper bounds of zeros of $M_{1}(h), M_{2}(h)$ and $M_{3}(h)$ in this example are strictly less than the corresponding upper bounds $K$ in Theorem 14. In the following we give proof of the conclusion.

Proof. From Theorem 2, we can check that system (41) is a Hamiltonian system with a global center if $|\epsilon|=0$. And $H(x, y)=2 x^{2}+y^{4}$, which is $(2,1)$-quasi-homogeneous with weight degree 4 and $\omega=p(x, y) d x+$ $q(x, y) d y$, where

$$
p(x, y)=\sum_{0 \leq i+j \leq 2} a_{i j} x^{i} y^{j}, q(x, y)=\sum_{0 \leq i+j \leq 2} b_{i j} x^{i} y^{j} .
$$

Let $x=r^{2} C s \theta, y=r \operatorname{Sn} \theta$, system (41) is transformed to

$$
d\left(r^{4}\right)-\epsilon \sum_{i=1}^{6}\left(r^{i-1} P_{i}(\theta) d r+r^{i} Q_{i}(\theta) d \theta\right)=0
$$

where

$$
\begin{gathered}
P_{1}=b_{00} S n \theta, \quad Q_{1}=b_{00} C s \theta \\
P_{2}=2 a_{00} C s \theta+b_{01} S n^{2} \theta, \quad Q_{2}=b_{01} C \operatorname{Cs} \theta \operatorname{Sn} \theta-a_{00} S n^{3} \theta \\
P_{3}=\left(2 a_{01}+b_{10}\right) C s \theta S n \theta+b_{02} S n^{3} \theta, Q_{3}=-a_{01} S n^{4} \theta+b_{10} C s^{2} \theta+b_{02} C s \theta S n^{2} \theta \\
P_{4}=\left(b_{11}+2 a_{02}\right) C s \theta S n^{2} \theta+2 a_{10} C s^{2} \theta \\
Q_{4}=b_{11} C s^{2} \theta S n \theta-a_{10} C s \theta S n^{3} \theta-a_{02} S n^{5} \theta \\
P_{5}=\left(2 a_{11}+b_{20}\right) C s^{2} \theta S n \theta, \quad Q_{5}=b_{20} C s^{3} \theta-a_{11} C s \theta S n^{4} \theta \\
P_{6}=2 a_{20} C s^{3} \theta, \quad Q_{6}=-a_{20} C s^{2} \theta S n^{3} \theta .
\end{gathered}
$$

It is easily calculated

$$
M_{1}(h)=\sum_{i=1}^{6} h^{\frac{i}{4}} \int_{0}^{\tau} Q_{i}(\theta) d \theta=m_{1,3} h^{\frac{3}{4}},
$$

where $m_{1,3}=\frac{b_{10}-a_{01}}{3} \tau$.

Thus, $M_{1}(h)$ has no zeros on $(0,+\infty)$. However, the upper bound $K$ of zeros of $M_{1}(h)$ in Theorem 14 is 2 . 
We now calculate Melnikov functions $M_{k}(h)(k \geq 2)$ by the algorithm in [2]. Let

$$
a_{1}(r, \theta)=a_{1,0}(\theta)+a_{1,1}(\theta) r
$$

where

$$
a_{1,0}(\theta)=\frac{2 a_{02}-b_{11}}{4} \int_{0}^{\theta} \operatorname{Snt} d t, a_{1,1}(\theta)=\frac{a_{11}-2 b_{20}}{4} \operatorname{Sn} \theta .
$$

It is easily checked $d \omega=d a_{1} \bigwedge d H$ when $M_{1}(h) \equiv 0$, so

$$
M_{2}(h)=\sum_{i=1}^{7} h^{\frac{i}{4}} \sum_{i=m+n} \int_{0}^{\tau} a_{1, m}(\theta) Q_{n}(\theta) d \theta=m_{2,1} h^{\frac{1}{4}}+m_{2,3} h^{\frac{3}{4}}+m_{2,5} h^{\frac{5}{4}}+m_{2,7} h^{\frac{7}{4}},
$$

where

$$
\begin{aligned}
& m_{2,1}=-\frac{\left(2 a_{02}-b_{11}\right) b_{00}}{4} \int_{0}^{\tau} S n^{2} \theta d \theta, \\
& m_{2,3}=-\frac{3 a_{00}\left(a_{11}-2 b_{20}\right)+b_{02}\left(2 a_{02}-b_{11}\right)}{36} \tau, \\
& m_{2,5}=\frac{5\left(a_{11}-2 b_{20}\right)\left(b_{11}-3 a_{02}\right)+\left(2 a_{02}-b_{11}\right)\left(3 a_{11}-11 b_{20}\right)}{100} \int_{0}^{\tau} S n^{2} \theta d \theta, \\
& m_{2,7}=-\frac{\left(a_{11}-2 b_{20}\right) a_{20}}{84} \tau .
\end{aligned}
$$

Therefore, $M_{2}(h)$ has at most 3 zeros on $(0,+\infty)$ and there exist parameters values such that $M_{2}(h)$ has exactly 3 zeros, which is strictly less than the upper bound $K=4$ of zeros of $M_{2}(h)$ in Theorem 14 .

If $M_{1}(h)=M_{2}(h) \equiv 0$, then we can obtain the four irreducible algebraic components of parameters space $\mathbb{R}^{12}$ as follows.

$$
\begin{aligned}
\left(C_{1}\right): & b_{10}=a_{01}, b_{11}=2 a_{02}, b_{20}=\frac{1}{2} a_{11} \\
\left(C_{2}\right): & b_{10}=a_{01}, a_{00}=a_{20}=a_{02}=b_{11}=0 ; \\
\left(C_{3}\right): & b_{10}=a_{01}, a_{11}=b_{00}=b_{20}=b_{02}=0 ; \\
\left(C_{4}\right): & b_{10}=a_{01}, a_{20}=b_{00}=0,3 a_{00}\left(a_{11}-2 b_{20}\right)+b_{02}\left(2 a_{02}-b_{11}\right)=0, \\
& 5\left(a_{11}-2 b_{20}\right)\left(b_{11}-3 a_{02}\right)+\left(2 a_{02}-b_{11}\right)\left(3 a_{11}-11 b_{20}\right)=0 .
\end{aligned}
$$

If parameters are in the irreducible algebraic component $C_{1}$, then system (41) is a Hamiltonian system with Hamiltonian

$$
H(x, y)=2 x^{2}+y^{4}-\epsilon\left(a_{00} x+b_{00} y+\frac{a_{01}}{2} x^{2}+a_{01} x y+\frac{b_{01}}{2} y^{2}+\frac{a_{20}}{3} x^{3}+\frac{a_{11}}{2} x^{2} y+a_{02} x y^{2}+\frac{b_{02}}{3} y^{3}\right) \text {. }
$$

It follows that $M_{k}(h) \equiv 0$ on $(0,+\infty)$ for any $k \geq 3$.

If parameters are in the irreducible algebraic component $C_{2}$, then we consider

$$
a_{2}(r, \theta)=a_{2,0}(\theta)+a_{2,1}(\theta) r+a_{2,2}(\theta) r^{2}
$$


where

$$
\begin{aligned}
& a_{2,0}=\frac{\left(a_{11}-2 b_{20}\right) a_{01}}{16} \int_{0}^{\theta} S n t d t, \\
& a_{2,1}=\frac{\left(a_{11}-2 b_{20}\right) a_{10}}{16} \operatorname{Sn} \theta, \\
& a_{2,2}=\frac{\left(a_{11}-2 b_{20}\right)\left(a_{11}-b_{20}\right)}{16} S n^{2} \theta .
\end{aligned}
$$

It is easily checked $d\left(a_{1} \omega\right)=d a_{2} \bigwedge d H$. Then

$$
M_{3}(h)=\sum_{i=1}^{9} h^{\frac{i}{4}} \sum_{i=m+n} \int_{0}^{\tau} a_{2, m}(\theta) Q_{n}(\theta) d \theta=m_{3,1} h^{\frac{1}{4}}+m_{3,3} h^{\frac{3}{4}}+m_{3,5} h^{\frac{5}{4}} .
$$

where

$$
\begin{aligned}
& m_{3,1}=-\frac{\left(a_{11}-2 b_{20}\right) a_{01} b_{00}}{16} \int_{0}^{\tau} S n^{2} \theta d \theta \\
& m_{3,3}=-\frac{\left(a_{11}-2 b_{20}\right) a_{01} b_{02}}{144} \tau \\
& m_{3,5}=-\frac{\left(a_{11}-2 b_{20}\right)\left(7 a_{11}+b_{20}\right) a_{01}}{400} \int_{0}^{\tau} S n^{2} \theta d \theta
\end{aligned}
$$

That shows $M_{3}(h)$ in $C_{2}$ has at most 2 zeros on $(0,+\infty)$, which is strictly less than the upper bound $K=7$ of zeros of $M_{3}(h)$ in Theorem 14.

Using the similar arguments, we can calculate $M_{3}(h)$ in the component $C_{3}$.

$$
M_{3}(h)=m_{3,1} h^{\frac{1}{4}}+m_{3,3} h^{\frac{3}{4}}+m_{3,5} h^{\frac{5}{4}},
$$

where

$$
\begin{aligned}
& m_{3,1}=-\frac{\left(2 a_{02}-b_{11}\right) a_{00} a_{01}}{16} \int_{0}^{\tau} S n^{2} \theta d \theta, \\
& m_{3,3}=-\frac{\left(2 a_{02}-b_{11}\right)\left(a_{02}+b_{11}\right) a_{01}}{144} \tau, \\
& m_{3,5}=-\frac{\left(2 a_{02}-b_{11}\right) a_{01} a_{20}}{80} \int_{0}^{\tau} S n^{2} \theta d \theta .
\end{aligned}
$$

And $M_{3}(h)$ in the component $C_{4}$,

$$
M_{3}(h)=m_{3,1} h^{\frac{1}{4}}+m_{3,3} h^{\frac{3}{4}}+m_{3,5} h^{\frac{5}{4}},
$$


where

$$
\begin{aligned}
m_{3,1}= & -\frac{\left(2 a_{02}-b_{11}\right)\left(3 a_{00} a_{01}+b_{01} b_{02}\right)}{48} \int_{0}^{\tau} S n^{2} \theta d \theta \\
m_{3,3}= & \frac{1}{1296}\left(\left(a_{11}-2 b_{20}\right)\left(-9 a_{01} b_{02}+2 b_{01} b_{11}\right)+\right. \\
& \left.\left(2 a_{02}-b_{11}\right)\left(-9 a_{01} a_{02}+9 a_{10} b_{02}-9 a_{01} b_{11}+4 b_{01} b_{20}\right)\right) \tau, \\
m_{3,5}= & -\frac{\left(a_{11}-2 b_{20}\right)\left(7 a_{11}+b_{20}\right) a_{01}}{400} \int_{0}^{\tau} S n^{2} \theta d \theta .
\end{aligned}
$$

So $M_{3}(h)$ has at most 2 zeros on $(0,+\infty)$ if parameters are in the irreducible algebraic components $C_{3}$ and $C_{4}$. We complete the proof.

\section{General Discussion and the Bogdanov-Takens HAMILTONIAN}

We explain how our method can be used in principle for any polynomial Hamiltonian which displays an isolated singularity of center type. We can assume that this center is $O=(0,0)$. Let recall an important theorem of singularity theory due to K. Saito (cf. [15]). Let $H$ be a germ of analytic function in $\left(\mathbb{K}^{N}, 0\right), \mathbb{K}=\mathbb{R}, \mathbb{C}$ which displays an isolated singularity at the origin $(0,0)$. Let $J$ be its Jacobian ideal (generated by the partial derivatives of $H$ ). Assume that $H$ belongs to its Jacobian ideal. Then there exists an analytic coordinate system $\left(x_{1}, \ldots, x_{N}\right), x(0)=0$ so that $H$ is quasi-homogeneous in the coordinates $x$ in the sense there exist rational positive numbers $\left(m_{1}, \ldots, m_{N}\right)$ so that the linear vector field $C=\sum_{i=1}^{N} m_{i} x_{i} \frac{\partial}{\partial x_{i}}$, satisfies $C . H=H$. In this article we restrict to dimension $N=2$ and write coordinates $(x, y)$.

In case $H$ has an isolated singularity at $(0,0)$, one can show (cf. $[3,6])$ that it always exists a polynomial $m(H)$ which belongs to the Jacobian ideal generated by $\left(\frac{\partial f}{\partial x}, \frac{\partial f}{\partial y}\right)$. More precisely, the quotient of the ring $K(x, y) / J$ is a finite dimensional vector space of dimension equal to the Milnor number $\mu$. The multiplication by $H$ induces a linear endomorphism of $K(x, y) / J \rightarrow K(x, y) / J$. The polynomial $m(H)$ can be choosen as the minimal polynomial of this endomorphism.

This means that there exists a polynomial vector field $C$ so that for any polynomial 1-form $\omega$ with $d \omega=D(x, y) d x \wedge d y$, the 1-form $\omega_{1}=\frac{1}{m(H)} D(x, y) \iota_{C} d x \wedge d y$ defines a Gelfand-Leray form for $\omega$ (meaning $\left.d \omega=\omega_{1} \wedge d H\right)$. Then we can, in principle, proceed with the algorithm 
of the successive derivatives of $[2,3,6]$ as we have explicit representation of the Gelfand-Leray derivative. In particular, this covers the case of the elliptic Bogdanov-Takens Hamiltonian:

$$
H=\frac{1}{2} y^{2}+\frac{1}{2} x^{2}-\frac{1}{3} x^{3}
$$

which was considered by Iliev ( $[10])$. This Hamiltonian displays a center at $(0,0)$ contained in a periodic annulus bounded by a homoclinic loop passing by the saddle $(0,1)$. We consider a perturbation of this Hamiltonian by a polynomial form of degree $n \geq 3$.

For instance, in the case of the elliptic Bogdanov-Takens Hamiltonian, there are two distinct critical values 0 and $\frac{1}{6}$; we can check that $m(H)=H\left(H-\frac{1}{6}\right)$. The Jacobian ideal is generated by $\left(y, x-x^{2}\right)$. It can be checked that there exists a polynomial vector field

$$
C=f(x) \frac{\partial}{\partial x}+g(x, y) \frac{\partial}{\partial y}
$$

of degree equal to 4 such that $C . H=m(H)$. Indeed, this displays:

$$
\begin{gathered}
H\left(H-\frac{1}{6}\right)=\left(\frac{1}{2} y^{2}+\frac{1}{2} x^{2}-\frac{1}{3} x^{3}\right)\left(\frac{1}{2} y^{2}+\frac{1}{2} x^{2}-\frac{1}{3} x^{3}-\frac{1}{6}\right) \\
m(H)=\frac{1}{4} y^{4}+\frac{1}{2} y^{2}\left[x^{2}-\frac{2}{3} x^{3}-\frac{1}{6}\right]+\left(\frac{1}{2} x^{2}-\frac{1}{3} x^{3}\right)\left(\frac{1}{2} x^{2}-\frac{1}{3} x^{3}-\frac{1}{6}\right) \\
m(H)=g(x, y) \frac{\partial H}{\partial y}+\left(\frac{1}{2} x^{2}-\frac{1}{3} x^{3}\right)\left(\frac{1}{2} x^{2}-\frac{1}{3} x^{3}-\frac{1}{6}\right),
\end{gathered}
$$

with $g(x, y)=\frac{1}{4} y^{3}+\frac{1}{2} y\left[x^{2}-\frac{2}{3} x^{3}-\frac{1}{6}\right]$ then,

$$
\left(\frac{1}{2} x^{2}-\frac{1}{3} x^{3}\right)\left(\frac{1}{2} x^{2}-\frac{1}{3} x^{3}-\frac{1}{6}\right)=f(x) \frac{\partial H}{\partial x},
$$

with $f(x)=x\left(\frac{1}{2}-\frac{1}{3} x\right)\left(\frac{1}{3} x^{2}-\frac{1}{6} x+\frac{1}{6}\right)$. So if we use this vector field $C$ to compute the Gelfand-Leray derivative $\omega_{1}$, we see that if $\omega$ is polynomial of degree less or equal than $n$, then $\omega_{1}$ is of the form $\frac{1}{m(H)} \bar{\omega}_{1}$, with $\bar{\omega}_{1}$ polynomial of degree less or equal than $n+3$.

Remark 18. Another example where the Gelfand-Leray derivative can be explicited after a global linearization is given in [5].

Finally, we observe that if we can obtain a bound on the number of isolated zeros of the bifurcation function $M_{k}(h)$ at any order $k$ and if there is a Bautin ideal associated to the perturbation scheme, the theorem 5 of the article [4] implies that the order $k$ is bounded. This 
solves the Arnol'd-Hilbert's 16th problem to any order for this class of perturbations.

\section{ACKNOWLEDGEMEnTS}

We warmly thank to the reviewer his/her comments and suggestions which help us to improve this paper.

\section{REFERENCES}

[1] A. Buica, J. Giné, J. Llibre. Bifurcation of limit cycles from a polynomial degenerate center. Adv. Nonlinear Stud. 10 (2010), no. 3, 597 - 609.

[2] J.-P. Françoise. Successive derivatives of a first return map, application to the study of quadratic vector fields. Ergodic Theory Dynam. Syst., 16(1996).

[3] J.-P. Françoise, M. Pelletier. Iterated integrals, Gelfand-Leray residue, and first return mapping. J. Dyn. Control Syst. 12 (2006), no. 3, 357 - 369.

[4] J.-P. Françoise, L. Gavrilov, D. Xiao. Hilbert's 16th problem on a period annulus and Nash space of arcs. Math. Proc. Cam. Phil. Soc. (2020), 1-33.

[5] J.-P. Françoise, D. Xiao. Perturbation theory of a symmetric center within Liénard equations. J. Differential Equations 259 (2015), no. 6, 2408 - 2429.

[6] L. Gavrilov. Higher order Poincare-Pontryagin functions and iterated path integrals. Ann.fac.sci.toulouse Math XIV(2005):663 - 682.

[7] L. Gavrilov, I. Iliev. Cubic perturbations of elliptic Hamiltonian vector fields of degree three. J. Differential Equations 260 (2016), 3963 - 3990.

[8] L. Gavrilov; J. Giné; M. Grau. On the cyclicity of weight-homogeneous centers. J. Differential Equations 246 (2009), no. 8, 3126 - 3135.

[9] I.D. Iliev. The number of limit cycles due to polynomial perturbations of the harmonic oscillator. Math. Proc. Cambridge Philos. Soc. 127 (1999), no. 2, $317-322$.

[10] I.D.Iliev. On the limit cycles available from polynomial perturbations of the Bogdanov-Takens Hamiltonian. Israel J. Math. 115 (2000), 269 - 284.

[11] Liapunov, A.M. Stability of motion. In: Mathematics in Science and Engineering, vol. 30. Academic Press, San Diego (1966)

[12] A. Jebrane, P. Mardesic, M. Pelletier. A generalization of Françoise's algorithm for calculating higher order Melnikov functions. Bull. Sci. math., 126(2002) $705-732$.

[13] A. Jebrane and H. Zoladek. A note on higher order Melnikov functions. Qualitative Theory of Dynamical Systems 6.2(2005):273 - 287.

[14] W. Li, J. Llibre, J. Yang and Z. Zhang. Limit cycles bifurcating from the period annulus of quasi-homogeneous centers. J. Dynamics and Differential Equations 21 (2009), 133 - 152.

[15] K. Saito. Quasihomogene isolierte Singularitäten von Hyperflächen. Invent. Math. 14 (1971), 123 - 142.

[16] P. Yang and J. Yu. The number of limit cycles from a cubic center by the Melnikov function of any order. J. Differential Equations 268 (2020), 1463-1494. 
[17] Z. Zhang and B. Li . High order Melnikov functions and the problem of uniformity in global bifurcation. Annali di Matematica pura ed applicata, CLXI (1992), $181-212$.

${ }^{1}$ Sorbonne Université, Laboratoire Jacques-Louis Lions, UMR 7598 CNRS, 4 Place Jussieu, 75252, Paris Cedex, France

Email address: Jean-Pierre.Francoise@upmc.fr

2 School of Mathematical Sciences, MOE-LSC, Shanghai Jiao Tong University, Shanghai 200240, China

Email address: hehongjin000@126.com

Email address: xiaodm@sjtu.edu.cn 